\title{
Effect of Prandtl Number on Forced Convection in a Two Sided Open Enclosure Using Nanofluid
}

\author{
S. Parvin", R. Nasrin, M. A. Alim, and N. F. Hossain \\ Department of Mathematics, Bangladesh University of Engineering and Technology, Dhaka-1000, \\ Bangladesh
}

Received 2 February 2012, accepted in final revised form 11 September 2012

\begin{abstract}
Analysis of Prandtl number effect on forced convective flow and thermal field characteristics inside an open cavity with porous wavy isothermal wall using water-CuO nanofluid have been performed numerically. The upper and lower surfaces are of temperature $T_{h}$. The fluid enters from left and exits from right with initial velocity $U_{i}$ and temperature $T_{i}$. Governing equations are discretized using the Finite Element Method. Simulation is carried out for a range of Prandtl number, $\operatorname{Pr}$ (from 1.47 to 8.81) and wave amplitude $A$ (from 0 to 0.15 ) while Reynolds number, $R e=100$; Darcy number, $D a=100$ and solid volume fraction, $\phi=5 \%$. Results are presented in the form of streamlines, isothermal lines, rate of heat transfer, average temperature of the fluid and velocity at midheight of the channel for various $\operatorname{Pr}$ and $A$. Increasing $\operatorname{Pr}$ and lessening $A$ causes the enhancement of heat transfer rate.
\end{abstract}

Keywords: Water-CuO nanofluid, forced convection, wavy enclosure, porous medium, finite element method.

(C) 2013 JSR Publications. ISSN: 2070-0237 (Print); 2070-0245 (Online). All rights reserved.

doi: http://dx.doi.org/10.3329/jsr.v5i1.9641 J. Sci. Res. 5 (1), $67-75$ (2013)

\section{Introduction}

Nanofluid technology has emerged as a new enhanced heat transfer technique in recent years. Nanofluid is made by adding nanoparticles and a surfactant into a base fluid can greatly enhance thermal conductivity and convective heat transfer. The diameters of nanoparticles are usually less than $100 \mathrm{~nm}$ which improves their suspension properties. The knowledge of free or forced convection heat transfer inside geometries of irregular shape (for example, wavy channel and pipe bend) for porous media has many significant engineering applications; for example, geothermal engineering, solar-collectors, performance of cold storage, and thermal insulation of buildings.

A considerable number of published articles are available that deal with flow characteristics, heat transfer, flow and heat transfer instability, transition to turbulence,

\footnotetext{
* Corresponding author: salpar@math.buet.ac.bd
} 
design aspects, etc. Significant contributions have been made by several researchers [1-3] in order to model the problems of this specific group. For wavy cavities filled with porous medium that obeys the Darcy law, Kumar and Shalini [4] reported flow and heat transfer results in a cavity with wavy bottom wall. Misirlioglu et al. [5] was analyzed free convection in a wavy cavity filled with a porous medium. For non-Darcy porous medium, Kumar and Gupta [6] reported the flow and thermal fields' characteristics in wavy cavities.

In the present investigation, non-Darcy natural convection is investigated inside a wavy enclosure at different surface waviness ratio, aspect ratio, and Darcy number. Kumar et al. [7] found the significant heat transfer enhancement by the dispersion of nanoparticles in the base fluid. Santra et al. [8] modelled the nanofluids as a nonNewtonian fluid and observed a systematic decrease of the heat transfer as the volume fraction of the nanofluids increased. Most of the published papers are concerned with the analysis of natural convection heat transfer of nanofluids in square or rectangular enclosures; for example, Ghasemi and Aminossadati [9] and Muthtamilselvan et al. [10]. Dai et al. [11] investigated convective flow drag and heat transfer of $\mathrm{CuO}$ nanofluid in a small tube. Their results showed that the pressure drop of the nanofluid per unit length was greater than that of water. Pfautsch [12] studied the characteristics, flow development, and heat transfer coefficient of nanofluids under laminar forced convection over a flat plate. He concluded that a significant increase in the heat transfer coefficient, about a $16 \%$ increase in the heat transfer coefficient for the water based nanofluid and about a $100 \%$ increase for the ethylene glycol based nanofluid.

The main issue discussed in this paper is the Prandtl number effect on forced convective flow and heat transfer characteristics of water- $\mathrm{CuO}$ nanofluid in the open enclosure.

\section{Physical Configuration}

Fig. 1 shows a schematic diagram of the wavy channel. The model describes a channel with two isothermal wavy walls of temperature $T_{h}$. Flow enters from left and leaves from the right. The inlet fluid velocity and temperature are $U_{i}$ and $T_{i}$ respectivly. The working fluid through the channel is water-based nanofluid containing $\mathrm{CuO}$ nanoparticles.

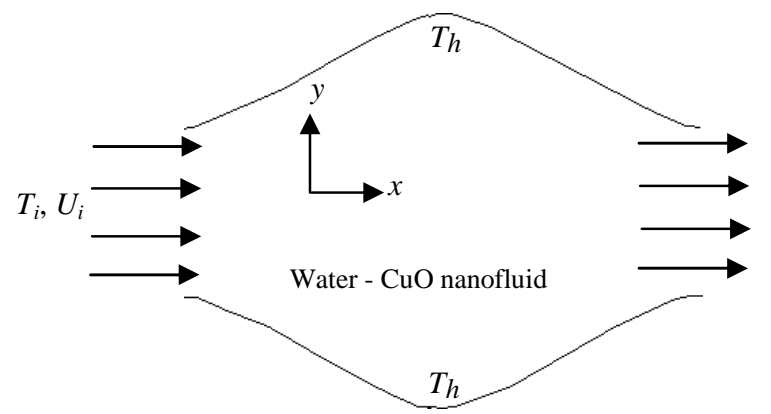

Fig. 1. Depiction of the geometry and the operation of the channel. 


\section{Mathematical Formulation}

In the present problem, it is considered that the flow is steady, two-dimensional, laminar, incompressible and there is no viscous dissipation. The radiation effect is neglected. The governing equations under Boussinesq approximation are as follows [13-15]:

$$
\begin{aligned}
& \frac{\partial u}{\partial x}+\frac{\partial v}{\partial y}=0 \\
& \rho_{n f}\left(\frac{\partial u}{\partial t}+u \frac{\partial u}{\partial x}+v \frac{\partial u}{\partial y}\right)=-\frac{\partial p}{\partial x}+\mu_{n f}\left(\frac{\partial^{2} u}{\partial x^{2}}+\frac{\partial^{2} u}{\partial y^{2}}\right)-\frac{\mu_{n f}}{K} u \\
& \rho_{n f}\left(\frac{\partial v}{\partial t}+u \frac{\partial v}{\partial x}+v \frac{\partial v}{\partial y}\right)=-\frac{\partial p}{\partial y}+\mu_{n f}\left(\frac{\partial^{2} v}{\partial x^{2}}+\frac{\partial^{2} v}{\partial y^{2}}\right)-\frac{\mu_{n f}}{K} v \\
& \frac{\partial T}{\partial t}+u \frac{\partial T}{\partial x}+v \frac{\partial T}{\partial y}=\alpha_{n f}\left(\frac{\partial^{2} T}{\partial x^{2}}+\frac{\partial^{2} T}{\partial y^{2}}\right)
\end{aligned}
$$

where, $\rho_{n f}=(1-\phi) \rho_{f}+\phi \rho_{s}$ is the density,

$\left(\rho C_{p}\right)_{n f}=(1-\phi)\left(\rho C_{p}\right)_{f}+\phi\left(\rho C_{p}\right)_{s}$ is the heat capacitance,

$\beta_{n f}=(1-\phi) \beta_{f}+\phi \beta_{s}$ is the thermal expansion coefficient,

$\alpha_{n f}=k_{n f} /\left(\rho C_{p}\right)_{n f}$ is the thermal diffusivity,

$\mu_{n f}=\mu_{f}(1-\phi)^{-2.5}$ is dynamic viscosity and

$k_{n f}=k_{f} \frac{k_{s}+2 k_{f}-2 \phi\left(k_{f}-k_{s}\right)}{k_{s}+2 k_{f}+\phi\left(k_{f}-k_{s}\right)}$ is the thermal conductivity of the nanofluid.

The boundary conditions are

at the upper and lower surfaces: $T=T_{h}$

at the inlet opening: $T=T_{i}, u=U_{i}$

at all solid boundaries: $u=v=0$

at the outlet opening convective boundary condition: $p=0$

The non-dimensionalized quantities used in this study are $X=\frac{x}{L}, Y=\frac{y}{L}, U=\frac{u}{U_{i}}$, $V=\frac{v}{U_{i}}$, Prandtl number, $\operatorname{Pr}=\frac{v_{f}}{\alpha_{f}}$ and Reynolds number, $\operatorname{Re}=\frac{U_{i} L}{v_{f}}$, Darcy number, $D a=\frac{K}{L^{2}}$ and temperature $\theta=\frac{T-T_{i}}{T_{h}-T_{i}}$, the average Nusselt number $N u=-\frac{1}{S} \int_{0}^{S}\left(\frac{k_{n f}}{k_{f}}\right) \frac{\partial \theta}{\partial N} d S$ and the mean temperature, $\theta_{a v}=\int \theta d \bar{V} / \bar{V}$. Here $\frac{\partial \theta}{\partial N}=\sqrt{\left(\frac{\partial \theta}{\partial X}\right)^{2}+\left(\frac{\partial \theta}{\partial Y}\right)^{2}}$ and $S, N, \bar{V}$ are the non-dimensional length, coordinate along the heated surface and volume of the channel respectively. 


\section{Numerical Technique}

The Galerkin finite element method $[16,17]$ is used to solve the non-dimensional governing equations along with boundary conditions for the considered problem. The equation of continuity has been used as a constraint due to mass conservation and this restriction may be used to find the pressure distribution. The continuity equation is automatically fulfilled for large values of this penalty constraint. Then the velocity components $(U, V)$, and temperature $(\theta)$ are expanded using a basis set. The Galerkin finite element technique yields the subsequent nonlinear residual equations. Three points Gaussian quadrature is used to evaluate the integrals in these equations. The non-linear residual equations are solved using Newton-Raphson method to determine the coefficients of the expansions. The convergence of solutions is assumed when the relative error for each variable between consecutive iterations is recorded below the convergence criterion $\varepsilon$ such that $\left|\psi^{n+1}-\psi^{n}\right| \leq 10^{-4}$, where $n$ is the number of iteration and $\Psi$ is a function of $U, V$, and $\theta$. For numerical computation and post processing the software COMSOL Multiphysics and Tecplot are used.

\subsection{Mesh generation}

In finite element method, the mesh generation is the technique to subdivide a domain into a set of sub-domains, called finite elements, control volume etc. The discrete locations are defined by the numerical grid, at which the variables are to be calculated. It is basically a discrete representation of the geometric domain on which the problem is to be solved. The computational domains with irregular geometries by a collection of finite elements make the method a valuable practical tool for the solution of boundary value problems arising in various fields of engineering. Fig. 2 displays the finite element mesh of the present physical domain.

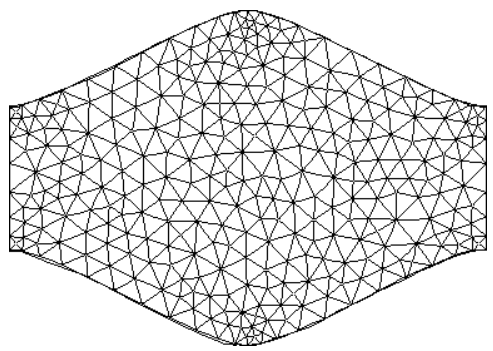

Fig. 2. Mesh generation of the channel.

\subsection{Grid refinement test}

In order to determine the proper grid size for this study, a grid independence test is conducted with five types of mesh for $\operatorname{Re}=100, \operatorname{Pr}=6.2, A=0.1, D a=100$ and $\phi=5 \%$. 
The extreme value of $N u$ is used as a sensitivity measure of the accuracy of the solution and is selected as the monitoring variable. Considering both the accuracy of numerical value and computational time, the present calculations are performed with 12666 nodes and 8657 elements grid system. This is described in Fig. 3.

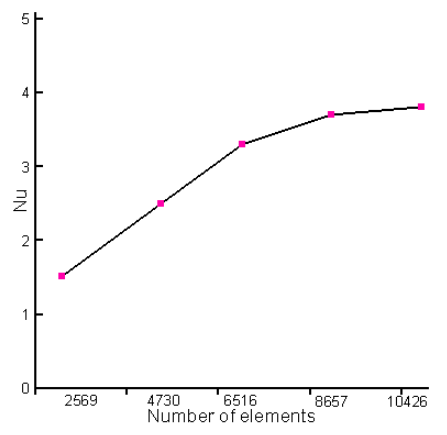

Fig. 3. Grid independency study for $D a=100, \operatorname{Re}=100, \operatorname{Pr}=6.2, A=0.1$ and $\phi=5 \%$.

\subsection{Thermo-physical properties}

The thermo-physical properties of fluid (water) and solid $\mathrm{CuO}$ are tabulated in Table 1. The properties are taken from [18].

Table 1. Thermo-physical properties of water-CuO nanofluid.

\begin{tabular}{ccc}
\hline Physical properties & Water & $\mathrm{CuO}$ \\
\hline$C_{p}$ & 4182 & 540 \\
$\rho$ & 998.1 & 6510 \\
$k$ & 0.6 & 18 \\
$\beta$ & $2.2 \times 10^{-4}$ & $8.5 \times 10^{-6}$ \\
\hline
\end{tabular}

\section{Results and Discussion}

In this section, numerical results in terms of streamlines and isotherms are displayed for various Prandtl number $\operatorname{Pr}(=1.47,3.7,6.2$ and 8.81$)$ and wave amplitude $A(=0,0.05$, 0.1 and 0.15 ) while $R e=100, D a=100$ and $\phi=5 \%$. In addition, the values of the average Nusselt number, average bulk temperature, mid-height horizontal velocity in the cavity have been calculated for water-CuO nanofluid.

The velocity (modulus of the velocity vector) field is displayed in Fig. 4(i). In the velocity vector, initially the flow covers the whole domain of the channel while it spreads from the middle of the channel due to increase viscous force from 1.47 to 8.81 . There is no significant change in streamlines except in the middle of the two sided open cavity. 
On the other hand, Fig. 4(ii) expresses the temperature field in terms of isothermal lines for different values of Prandtl number. This figure shows that at low values of Pr, the temperature of the nanofluid rapidly reaches to the temperature of hot walls due to low viscosity. With increasing Prandtl number, decrement of temperature of water-CuO nonofluid happens slowly which leads to increment of peaks of isothermal lines at the middle of the channel.

(ii)

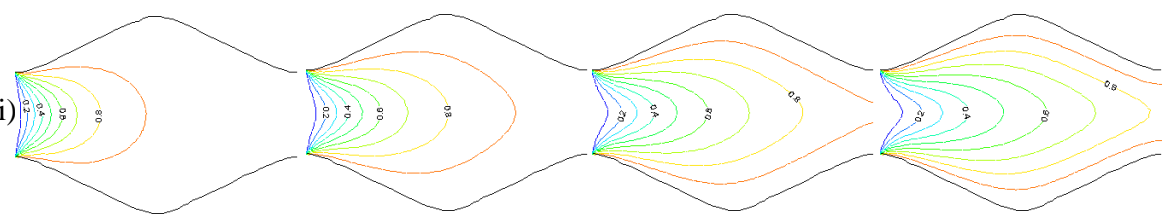

(i)

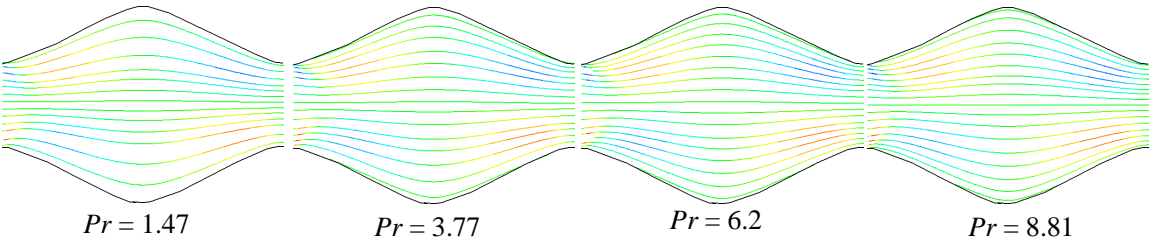

Fig. 4. Effect of $\operatorname{Pr}$ on (i) streamlines and (ii) isotherms at $A=0.1$.

Figs. 5(i)-(iii) show plot of the average heat transfer rate $(N u)$, average average bulk temperature $\left(\theta_{a v}\right)$ and mid-height $U$-velocity for different $\operatorname{Pr}$. The rate of heat transfer enhances for rising $\operatorname{Pr}$ in presence of nanoparticles. The mean bulk temperature $\left(\theta_{a v}\right)$ devalues for escalating $\mathrm{Pr}$. It is seen from Fig. 5(iii) that the wave amplitude for the lowest Prandtl number is found greater than the remaining values of this parameter. This is due to the fact that the increase of viscous force leads to retardation in the velocity profile.

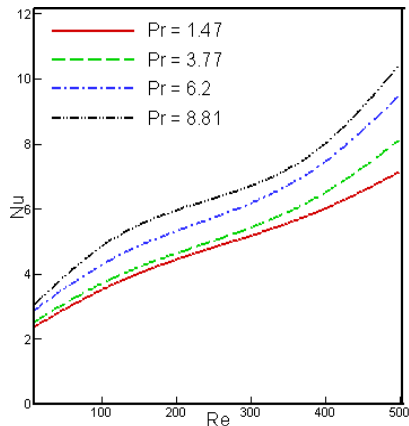

(i)

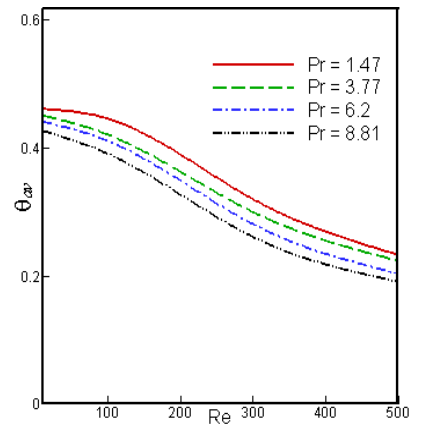

(ii)

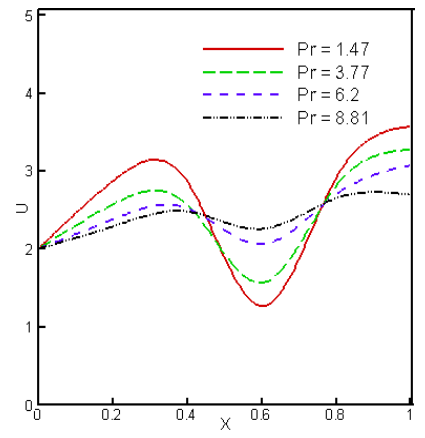

(iii)

Fig. 5. (i) rate of heat transfer, (ii) mean temperature and (iii) mid-height $U$-velocity for different $P r$. 
The effect of non-dimensional wave amplitude $A$ on the flow field is presented in Fig. 6 (i) while $\operatorname{Pr}=6.2$. The strength of the flow circulation and thermal current activities is much more activated with escalating $A$. In the velocity vector, initially the flow covers the whole domain of the channel while it concentrates near the middle part of the channel due to increase wave amplitude from 0 to 0.15 . With escalating $A$ the streamlines starts to take wavy pattern at the channel walls. This happens due to waviness of the walls.

Fig. 6 (ii) expresses the effect of non-dimensional wave amplitude $A$ on the thermal field at $\operatorname{Pr}=6.2$. The temperature lines through the horizontal corrugated pipe dense near the inlet for increasing $A$. But initially $(A=0)$ they are horizontal near the walls corresponding to physical changes of the current geometry. Due to rising values of wave amplitude, the temperature distributions become distorted resulting in an increase in the overall heat transfer. This result can be attributed to the performance of the corrugated surface. It is worth noting that as the wave amplitude increases, the thickness of the thermal boundary layer near the input opening enhances which indicates a steep temperature gradient and hence, an increase in the overall heat transfer from the corrugated wall to the cold water based $\mathrm{CuO}$ nanofluid. Isothermal lines show that at the highest value of $A(=0.15)$, the temperature of the nanofluid rapidly reaches to the temperature of hot walls.

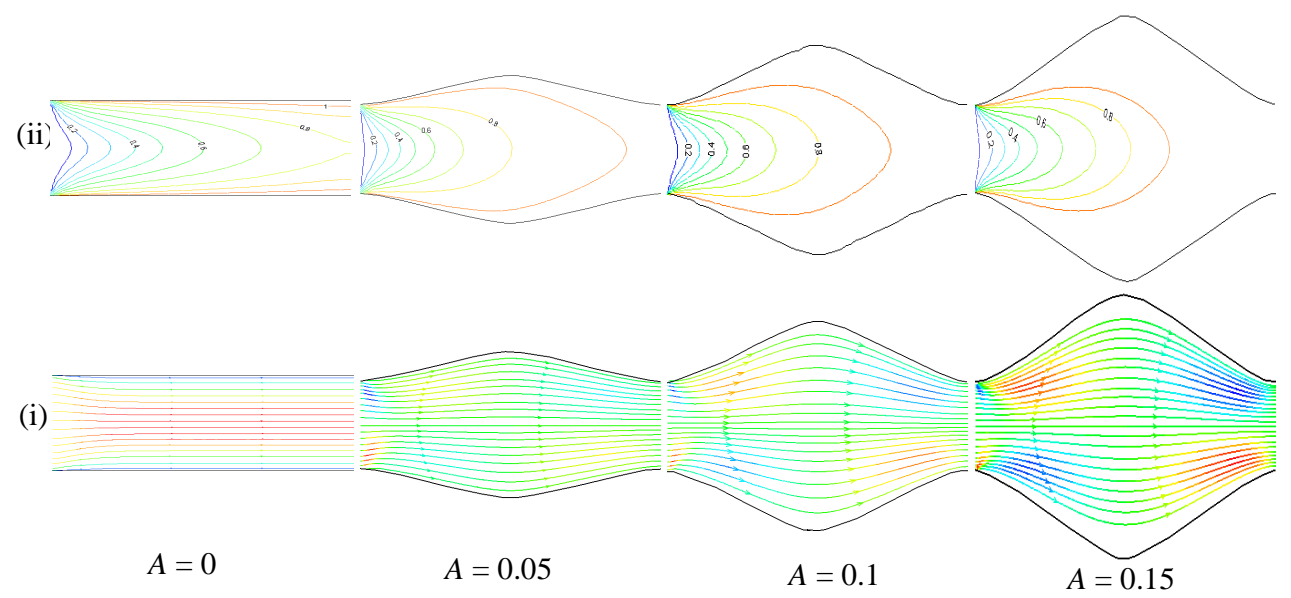

Fig. 6. Effect of $A$ on (i) streamlines and (ii) isotherms at $\operatorname{Pr}=6.2$.

The mean Nusselt number $(\mathrm{Nu})$, mean temperature $\left(\theta_{a v}\right)$ and mid-height horizontal velocity component for various $A$ are depicted in Fig. 7(i)-(iii). Increasing wave amplitude of the current geometry devalues average heat transfer rate. Fig. 7(ii) shows that the mean non-dimensional temperature grows up for the rising values of $A$. Consequently, the mid height $U$ velocity component reduces for higher wave amplitude. This is due to the fact that the increase of viscous force leads to retardation in the velocity profile. 


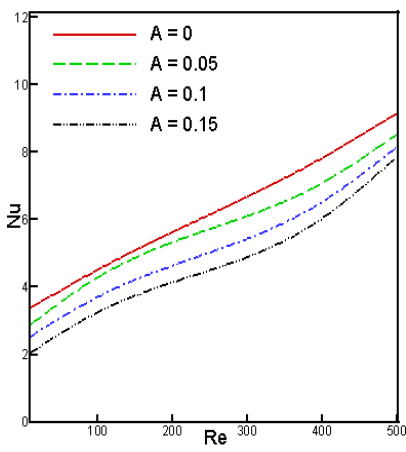

(i)

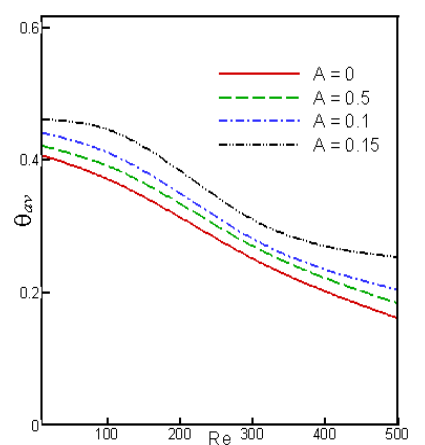

(ii)

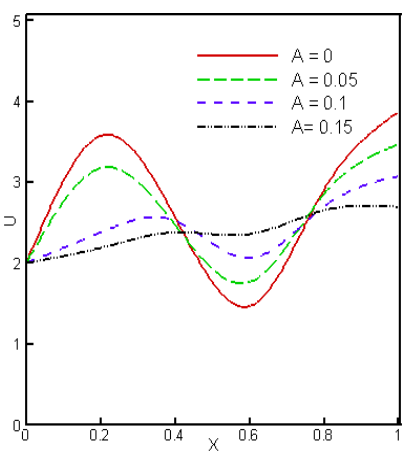

(iii)

Fig. 7. (i) Rate of heat transfer, (ii) mean temperature and (iii) mid-height $U$-velocity for different $A$.

\section{Conclusion}

The problem of the Prandtl number effect on forced convection heat transfer in a wavy open cavity filled with water-CuO nanofluid has been studied numerically. Flow and temperature field in terms of streamlines and isotherms have been displayed. The results of the numerical analysis lead to the following conclusions:

- The structure of the fluid flow and temperature field through the channel is found to be significantly dependent upon the Prandtl number and wave and amplitude.

- The maximum rate of heat transfer is obtained for the highest $\operatorname{Pr}$ and lowest $A$.

- The mean temperature of the fluid in the channel decrease with rising $\operatorname{Pr}$ and diminishing $A$.

- The mid height horizontal velocity profile is perturbed appreciably for the considered parameters.

\section{References}

1. L. Adjlout, O. Imine, A. Azzi, and M. Belkadi, Int. J. Heat Mass Trans. 45, 2141 (2002). http://dx.doi.org/10.1016/S0017-9310(01)00304-0

2. S. Mahmud and A.K.M.S. Islam, Int. J. Therm. Sci. 42, 1003 (2003). http://dx.doi.org/10.1016/S1290-0729(03)00076-0

3. P. K. Das and S. Mahmud, Int. J. Therm. Sci. 42, 397 (2003). http://dx.doi.org/10.1016/S1290-0729(02)00040-6

4. B. V. R. Kumar and Shalini, Num. Heat Trans. Part A 43, 753 (2003).

5. A. Misirlioglu and A.C. Baytas, I. Pop, Int. J. Heat Mass Trans. 48, 1840 (2005). http://dx.doi.org/10.1016/j.ijheatmasstransfer.2004.12.005

6. B. V. R. Kumar and S. Gupta, J. Porous Media 7, 261 (2004). http://dx.doi.org/10.1615/JPorMedia.v7.i4.20

7. S. Kumar and S. K. Prasad, J. Banerjee, App. Math. Model. 34, 573 (2009).

8. A. K. Santra and S. Sen, N. Chakraborty, J. Enhan. Heat Trans. 15 (4), 273 (2008).

9. B. Ghasemi and S. M. Aminossadati, Num. Heat Trans. Part A 55, 807 (2009). http://dx.doi.org/10.1080/10407780902864623 
10. M. Muthtamilselvan, P. Kandaswamy, and J. Lee, Commun. Nonlinear Sci. Num. Simul. 15 (6), 1501 (2009). http://dx.doi.org/10.1016/j.cnsns.2009.06.015

11. W. T. Dai, J. M. Li, X. Chen, and B. X. Wang, J. Eng. Thermophys. 24 (4), 633 (2003).

12. E. Pfautsch, Forced convection in nanofluids over a flat plate, Thesis Presented to the Faculty of the Graduate School, University of Missouri (2008).

13. R. Nasrin, J. Sci. Res. 3 (3), 501 (2011). http://dx.doi.org/10.3329/jsr.v3i3.7433

14. S. Parvin and R. Nasrin, J. Sci. Res. 4 (2), 337 (2012). http://dx.doi.org/10.3329/jsr.v4i2.8142

15. R. Nasrin, J. Sci. Res. 4 (1), 39 (2012). http://dx.doi.org/10.3329/jsr.v4i1.8014

16. C. Taylor and P. Hood, Computer and Fluids 1, 73 (1973). http://dx.doi.org/10.1016/0045-7930(73)90027-3

17. P. Dechaumphai, Finite Element Method in Engineering, 2nd ed. (Chulalongkorn University Press, Bangkok, 1999).

18. Zi-Tao Yu, Xu Xu, Ya-Cai Hu, Li-Wu Fan, and Ke-Fa Cen, Int. J. Heat Mass Trans. 54, 526 (2011). http://dx.doi.org/10.1016/j.ijheatmasstransfer.2010.09.017 